\title{
Temporal profile measurements of relativistic electron bunch based on wakefield generation
}

\author{
S. Bettoni, ${ }^{1}$ P. Craievich, ${ }^{1}$ A. A. Lutman, ${ }^{2}$ and M. Pedrozzi ${ }^{1}$ \\ ${ }^{1}$ Paul Scherrer Institut, 5232 Villigen, Switzerland \\ ${ }^{2}$ SLAC National Accelerator Laboratory, Menlo Park, California 94025, USA
}

(Received 24 September 2015; published 25 February 2016)

\begin{abstract}
A complete characterization of the time-resolved longitudinal beam phase space is important to optimize the final performances of an accelerator, and in particular this is crucial for Free Electron Laser (FEL) facilities. In this paper we propose a novel method to characterize the profile of a relativistic electron bunch by passively streaking the beam using its self-interaction with the transverse wakefield excited by the bunch itself passing off-axis through a dielectric-lined or a corrugated waveguide. Results of a proof-of-principle experiment at the SwissFEL Injector Test Facility are discussed.
\end{abstract}

DOI: 10.1103/PhysRevAccelBeams.19.021304

Several Free Electron Laser (FEL) facilities are in operation and other are being built to produce high power $\mathrm{X}$-ray coherent radiation, which is used for applications in physics, biology and material science. Very short bunches are essential to reach the time resolution required by these kinds of experiments. At the source relatively long bunches (from 1 to $10 \mathrm{ps}$ length) are typically generated to counteract the effect of the space charge forces at low energy. Only after acceleration the beams are compressed by two orders of magnitude in one or more stages. Several methods are used to retrieve the temporal duration and the shape of picosecond and sub-picosecond electron bunches. These methods are based on electro-optic sampling [1], coherent radiation $[2,3]$, streak cameras [4] or radio frequency (rf) transverse deflecting structures (TDS) [5-7]. TDS are the most used devices and provide a time-varying transversely deflecting field which induces a correlation between time and the transverse beam distribution. The longitudinal profile of the bunch is then reconstructed from the resulting image of the streaked beam on a downstream profile monitor. The electron beam energy can be time-resolved if a dipole magnet adds dispersion in the direction orthogonal to the one used for the streaking. Time-resolved measurements of the electron beams are crucial for X-ray FEL based on linear accelerators. Such measurements are routinely used as diagnostic to optimize the electron beam. Recently, the installation of an X-band TDS downstream of the undulator line at the Linac Coherent Light Source (LCLS) [8] allowed us to reconstruct the photon pulse shape from the timeresolved electron bunch energy losses [9]. The rf transverse deflecting structures become very expensive for high energy beams (several $\mathrm{GeV}$ ) and short bunch length (sub-ps),

Published by the American Physical Society under the terms of the Creative Commons Attribution 3.0 License. Further distribution of this work must maintain attribution to the author(s) and the published article's title, journal citation, and DOI. requiring high voltage and frequency to effectively streak the bunch. Shot-to-shot rf phase and electron bunch arrival time jitter translate to an orbit and a transverse position jitter downstream of the TDS and on the profile monitor. This orbit jitter limits applicable voltage at certain electron beam energies. Recently several possibilities have been considered to manipulate a relativistic beam using its interaction with the longitudinal wakefield excited by it passing in a dielectric-lined or a corrugated waveguide. They include the possibility to compensate for the energy chirp [10-12], and in general to linearize the longitudinal phase space beam distribution resulting from the bunch compression process $[13,14]$, and to drive coherent terahertz radiation inducing an energy modulation [15].

In this paper we propose a novel approach to perform time-resolved measurements of a relativistic electron bunch based on the self-transverse wakefield interaction of the beam itself passing off-axis through a dielectric-lined or corrugated waveguide. This device, the passive streaker, does not need to be powered and its manufacture is simpler compared to a rf TDS, resulting in a considerable cost saving. A passive streaker is self-synchronized with the beam by design, being the wakefield excited by the bunch itself. However, it cannot be easily calibrated as a rf TDS, by measuring the deflection of the centroid as function of the $\mathrm{rf}$ phase. The resolution in a passive streaker depends on the longitudinal coordinate on the electron bunch and it is intrinsically poorer on the bunch head. The reconstruction of the longitudinal beam profile involves then an iterative algorithm. In this paper we also present the first proof-of-principle experimental demonstration performed at the SwissFEL Injector Test Facility (SITF) [16] of a passive streaker based on a cylindrically symmetric dielectric-lined waveguide.

Figure 1 shows the schematic geometry of a dielectriclined waveguide which can be used as a passive streaker. Multi-mode and single-mode wakefield generation in a 


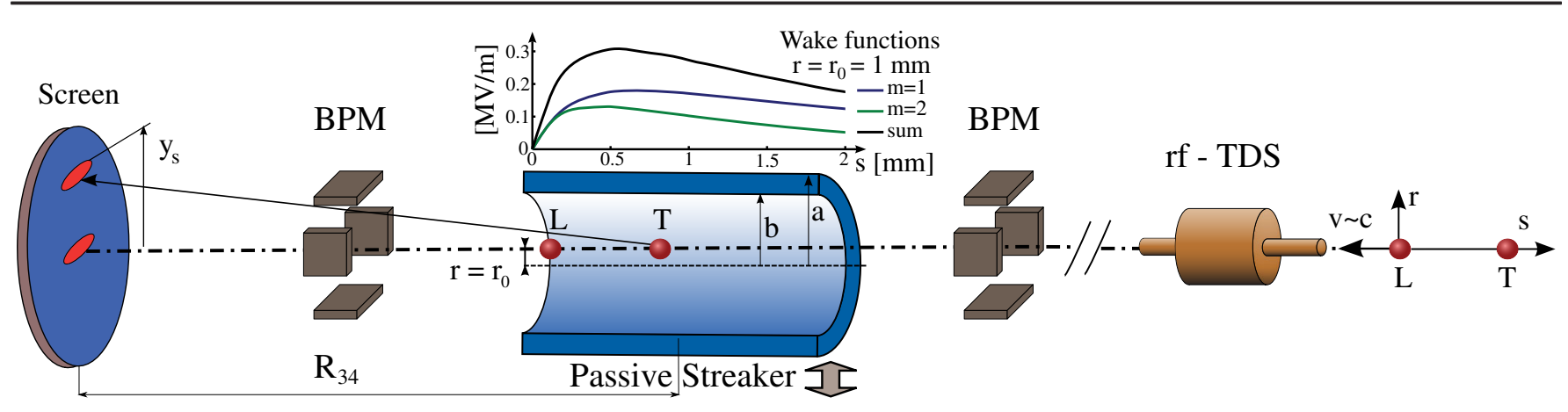

FIG. 1. Schematic layout of the dielectric-lined waveguide (passive streaker) used for the experiment at SITF. $L$ is the leading particle that travels along the cylindrical waveguide at an offset $r_{0}, T$ is the trailing particle with an offset $r$.

cylindrical symmetric dielectric-lined waveguide have been investigated in [17-23], respectively. Following [23], we summarize here the relevant aspects of transverse wakefield generated when a relativistic electron beam travels along a passive streaker at an offset $r_{0}$ from the axis. For the dielectric-lined waveguide, neglecting the transient effect at the entrance of the tube, the radial wake functions of the multipole with index $m$, when leading and trailing charge travel with the same angle $\varphi=\varphi_{0}$, are given by:

$$
w_{r, m}\left(r, s ; r_{0}\right)=\frac{Z_{0} c}{4 \pi a^{2}}\left(\frac{r_{0}}{a}\right)^{m}\left(\frac{r}{a}\right)^{m-1} \sum_{i=1}^{\infty} A_{m, i} \sin \left(k_{m, i} s\right),
$$

where $A_{m, i}$ is the dimensionless amplitude, $k_{m, i}$ is the wave number of the $i$ th eigenmode. $A_{m, i}$ and $\lambda_{m, i}$ depend on the dielectric constant $\epsilon$ and on the inner and the outer radius of the dielectric-lined waveguide $b$ and $a$, respectively. $Z_{0}$ and $c$ are the free-space impedance and the speed of light in vacuum. The transverse wake potentials $W_{r}\left(r, s ; r_{0}\right)$ of a bunch of charge distribution $\rho_{t}(r, \varphi) \rho_{l}(s)$ are calculated as the convolution of the distribution with the wake function, and the kick factor $K$ is given by the average transverse wake potential. The transverse kick imparts a transverse momentum on the bunch as a function of $s$ and of the transverse position of each particle. In the following we consider the dipole $(m=1)$ and the quadrupole $(m=2)$ contributions assuming a bunch with charge $Q$ and energy $E$ traveling off-axis along the passive streaker $\left(r_{0}, \varphi_{0}=90^{\circ}\right)$ with transverse size much smaller than $r_{0}$ $\left(\varphi \approx \varphi_{0}\right)$. The transverse displacement at the screen location, $y_{s}$, is then proportional to the angular-to-spatial transfer matrix element $R_{34}$ :

$$
y_{s}\left(r, r_{0}, s\right) \approx \frac{Q L_{p} R_{34}}{E}\left[W_{r, 1}\left(s ; r_{0}\right)+W_{r, 2}\left(r, s ; r_{0}\right)\right],
$$

where $W_{r, 1}$ and $W_{r, 2}$ are the dipole and the quadrupole wake potential, respectively. $W_{r, 1}$ does not depend on the trailing charge offset and $W_{r, 2}$ has a linear dependence on it.
Let $\rho_{s}\left(y_{s}\right)$ be the charge distribution at the screen then the equation $\rho_{s}\left(y_{s}\right) d y_{s}=\rho_{l}(s) d s$ is satisfied for the principle of charge conservation. Neglecting the transverse effects we can write

$$
\rho_{s}\left(y_{s}\right)=\rho_{l}\left[s\left(y_{s}\right)\right]\left|s^{\prime}\left(y_{s}\right)\right|,
$$

where $s^{\prime}\left(y_{s}\right)$ is the derivative of the inverse relation $s\left(y_{s}\right)$. We assume that the beam parameters besides the current profile at the streaker are independent of the longitudinal coordinate and that optics between the passive streaker and the screen is linear. With these assumptions the streaked profile on the screen is evaluated as the convolution of the measured normalized transverse profile when the beam transits the streaker on-axis $\rho_{0 s}\left(y_{s}\right)$ and the one derived by Eq. (3),

$$
\rho_{T s}\left(y_{s}\right)=\rho_{s}\left(y_{s}\right) \otimes \rho_{0 s}\left(y_{s}\right) .
$$

The calibration factor can be defined as the derivative of the transverse displacement at the screen in Eq. (2) as $S(s)=d y_{s}(s) / d s$. The resolution can be estimated from the vertical size of $\rho_{0 s}\left(y_{s}\right), \sigma_{y 0}$, and $S(s)$ as $\sigma_{s, R}(s) \geq \sigma_{y 0} / S(s)$. For an unambiguous reconstruction of the current profile, the wake potential along the electron bunch must be a strictly monotonic function of the beam longitudinal coordinate. The range over which this condition is fulfilled depends on the charge distribution, the properties of the dielectric layer and the geometry of the passive streaker. A reconstruction algorithm has been developed to time-resolve the electron beam current profile $\rho_{l}(s)$ from its trace on the downstream screen. The current profile of the electron bunch $\rho_{l}(s)$ is modeled by a nonnegative piecewise cubic polynomial whose knots and coefficients are iteratively optimized. The cost function is the difference between the measured profile at the screen and the one calculated from $\rho_{l}(s)$ and the measured $\rho_{0 s}\left(y_{s}\right)$ as in Eqs. (3) and (4). To this aim, the wake potential and its derivative are evaluated analytically as a function of the polynomial coefficients and the strictly monotonic wake potential is then inverted numerically. We extensively 


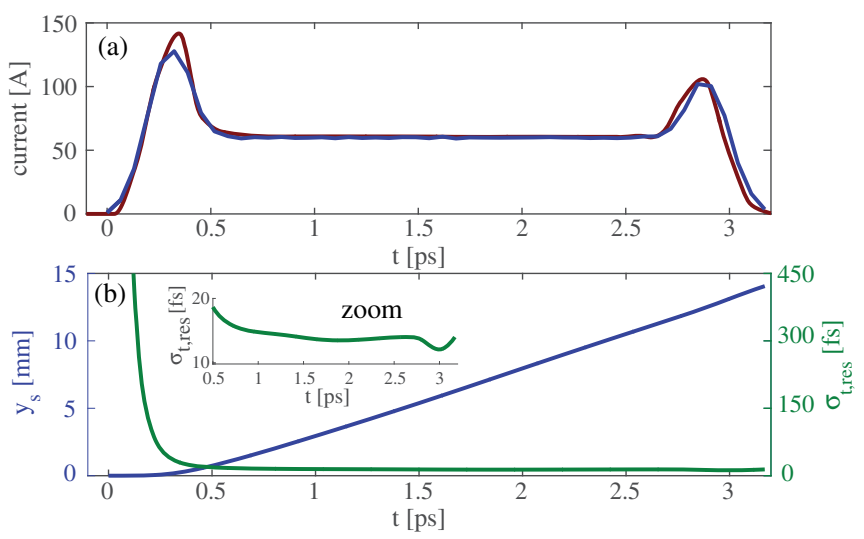

FIG. 2. (a) Initial bunch current distribution (blue) and reconstructed temporal profile (red). (b) Transverse displacement (blue) and resolution (green) at the screen as a function of the temporal coordinate.

tested the reconstruction algorithm assuming several initial beam distributions tracked using the numerical simulation code ElEGANT [24]. In Fig. 2(a) we show a current profile with a shape similar to the one typically obtained downstream of the undulator lines (see e.g., [9]) and the reconstructed one applying our algorithm. The inputs for the algorithm were the simulated transverse profiles of the beam at the screen when passing on-axis and off-axis in the passive streaker. This proves the validity of the reconstruction algorithm when the wake function is known and the wake potential is a monotonic function along the full electron bunch length. Figure 2(b) shows the transverse displacement $y_{s}$ and the resolution of the reconstruction. Considering a constant slope in the core of $y_{s}$ then the calibration factor is $S \approx 5 \mathrm{~mm} / \mathrm{ps}$. The time resolution in the core of the bunch for this specific example is $\sigma_{t, R} \geq 14$ fs with $\sigma_{y 0}=70 \mu \mathrm{m}$.

As a proof-of-principle experiment, we performed timeresolved measurements based on the passive deflection of a relativistic electron bunch at the end of the injector test facility. Figure 1 shows the schematic layout of the experiment. SITF is based on an S-band rf photo-cathode gun, four $4 \mathrm{~m}$ long S-band accelerating structures, which accelerate the beam and provide the chirp for the bunch compression. The beam is compressed via a magnetic bunch compressor, and a diagnostics section equipped with quadrupoles, a $0.5 \mathrm{~m}$ long rf TDS [25] and a spectrometer arm, where the longitudinal and transverse phase space of the beam are fully characterized. Two BPMs were used to align the beam through the passive streaker mounted on a support which allowed us to adjust vertically the position of the dielectric waveguide in steps of $10 \mu \mathrm{m}$. The transverse beam profiles of the streaked beam were imaged on a downstream YAG:Ce-type (Cerium-doped Yttrium aluminum garnet) screen. The dielectric-lined waveguide is $9.5 \mathrm{~cm}$ long $\left(\mathrm{L}_{p}\right)$ due to space limitations at SITF. It has an inner $(b)$ and outer $(a)$ radius of 1.65 and $2.40 \mathrm{~mm}$, respectively. The tube was made of alumina, chosen because of its relatively high dielectric constant $\epsilon=10$, necessary to obtain a monotonic wake function for picosecond bunch length. The external surface of the dielectric pipe has a $20 \mu \mathrm{m}$ copper coating, sufficient to shield the beam induced electric field. The experiment was performed with a $200 \mathrm{pC}, 140 \mathrm{MeV}$ electron bunch. The bunch peak current and the rms length after compression were $\sim 80 \mathrm{~A}$ and $0.9 \mathrm{ps}$, respectively. Streaked transverse images were measured at the YAG:Ce screen for different streaker offsets $\Delta y$ shown in Fig. 3(a-c). Figure 3(d) shows the comparison between the measured average vertical beam position (centroid) shift induced by the transverse wake as a function of $\Delta y$ and the one expected from the analytical model. The expected centroid displacement was calculated for the electron bunch current profile measured with the TDS. The dashed line shows that the dipole mode alone is insufficient to describe the transverse displacement for large offsets $\Delta y$. Instead, considering both dipole and quadrupole wakes (solid line), there is excellent agreement between the measurement and the model expectation. The linear and the cubic kick factors calculated from the model are $0.62 \mathrm{MV} /(\mathrm{nC} \mathrm{m} \mathrm{mm})$ and $0.52 \mathrm{MV} /\left(\mathrm{nC} \mathrm{m} \mathrm{mm}^{3}\right)$, respectively, whereas by fitting the experimental data with a cubic polynomial, the corresponding linear and cubic kick factors are $0.63 \mathrm{MV} /(\mathrm{nC} \mathrm{m} \mathrm{mm})$ and $0.43 \mathrm{MV} /\left(\mathrm{nC} \mathrm{m} \mathrm{mm}^{3}\right)$.
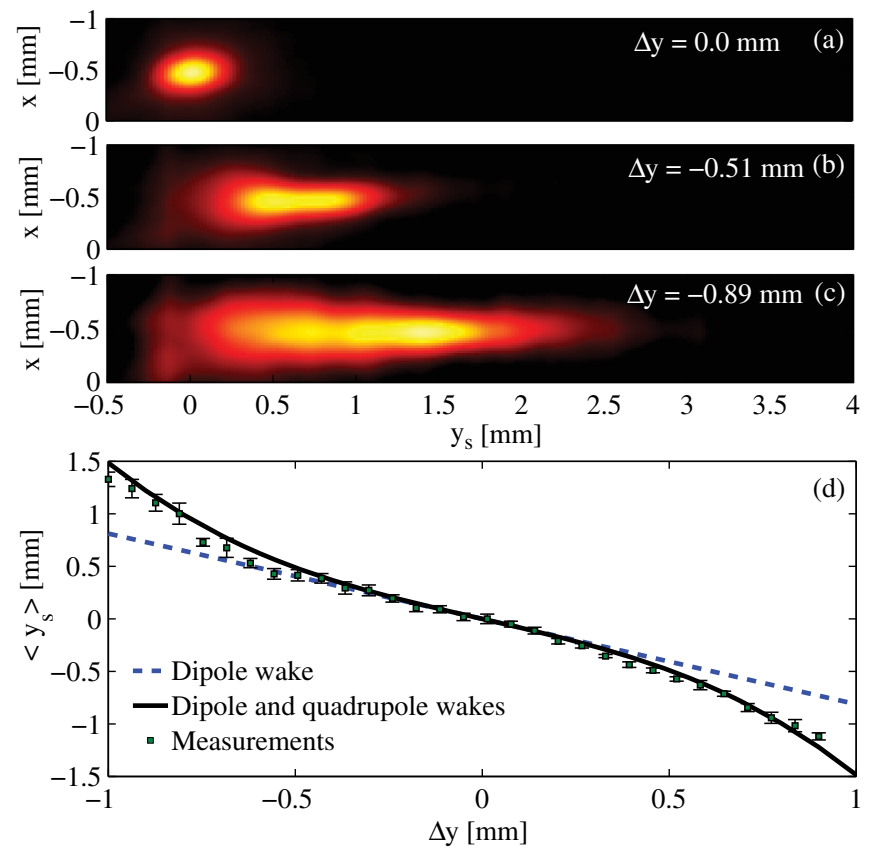

FIG. 3. (a-c) Measured transverse beam images at YAG:Ce screen for different offsets of the passive streaker. The head of the bunch is on the right and the tail on the left. (d) Measured average vertical beam centroid as a function of the vertical offset of the passive streaker against model expectations based on the dipole wake function (dashed) and the dipole and quadrupole wake functions (solid). 
We applied the algorithm successfully tested in simulations to the profile measured at the screen corresponding to the maximum offset shown in Fig. 3(c). The transverse beam dimension along the passive streaker, computed from the measured bunch size at the screen with the beam passing on axis in the passive streaker and the optics between the streaker and the screen, was $360 \mu \mathrm{m} \mathrm{rms}$. Such transverse size was too large to neglect the defocusing effect from the quadrupole wake. The charge distribution used for the convolution in Eq. (4), to include the defocusing effects for a transverse beam distribution at the streaker, around the offset $\Delta y, \rho_{\tau}(y)$, is

$$
\rho_{s}\left(y_{s}\right)=\int \rho_{s}\left(\tilde{y_{s}}\right) \rho_{\tau}\left[\frac{\Delta y\left(y_{s}-\tilde{y_{s}}\right)}{y_{s q}\left(\tilde{y_{s}}\right)}\right] \frac{\Delta y}{y_{s q}\left(\tilde{y}_{s}\right)} d \tilde{y_{s}},
$$

where $y_{s q}\left(y_{s}\right)$ is the transverse displacement at the screen due to the quadrupole wake only, for a particle at the offset $\Delta y$ at the tube that is deflected to the coordinate $y_{s}$ at the screen.

Figure 4(a) shows the comparison of the temporal beam current profile reconstructed applying the algorithm and the one measured using the TDS. The profile at the screen obtained by neglecting the transverse finite emittance effects, presents large oscillations where the resolution is
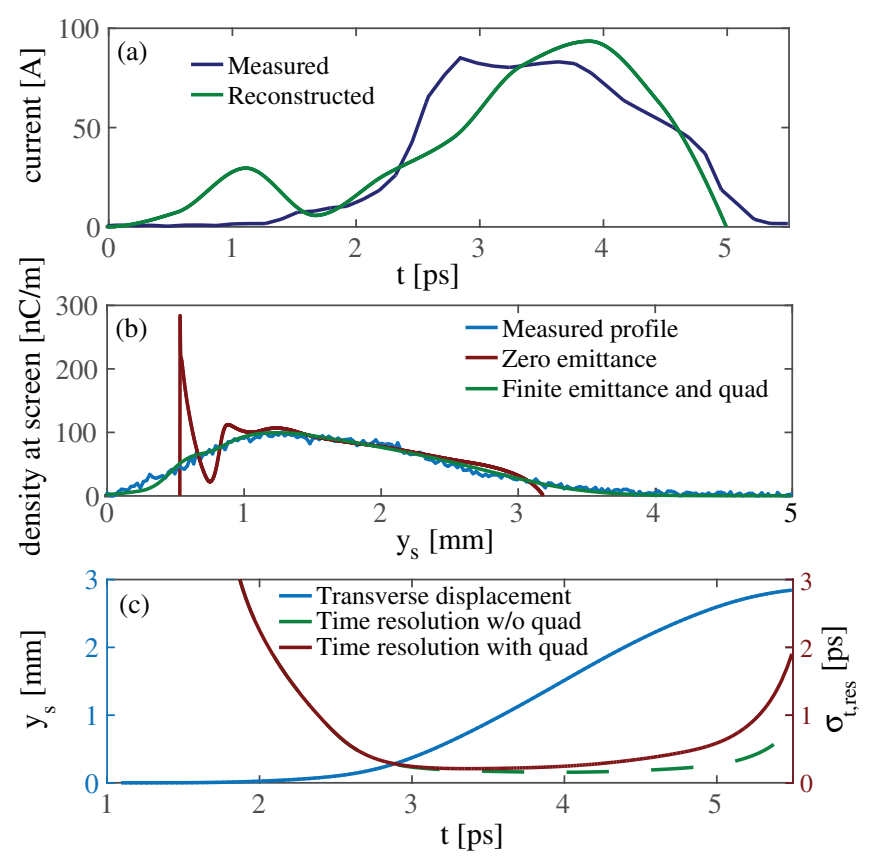

FIG. 4. (a) Temporal profile measured with the TDS (dashed). Temporal profile reconstructed from the passively streaked beam (solid). (b) Measured and reconstructed transverse profiles at the screen. (c) Left axis: transverse displacement at the screen calculated with Eq. (2) with offset $\Delta y=0.89 \mathrm{~mm}$ (blue). Right axis: temporal resolution without defocusing (dashed) and with defocusing (red) of the passive streaker against the longitudinal coordinate inside the bunch. poor, on the bunch head, and it is shorter than the measured one. Distribution details on the bunch head are lost with the convolution in Eq. (4) and could not be recovered in this proof-of-principle experiment. The temporal resolution, depicted in Fig. 4(c), is not constant along the longitudinal coordinate of the bunch but proportional to the derivative of the wake potential [see Eq. (3)], which in turn depends on the longitudinal position of the particles along the beam itself. A large transverse size at the passive streaker decreases the resolution also at the bunch tail, when the deflection due to the quadrupole wake is relevant.

In conclusion, the novel approach described in this paper provides an alternative method to time-resolve the longitudinal profile of a relativistic electron bunch. The method is based on the self-transverse-wakefield interaction of the electron bunch beam passing off-axis through a structure capable of generating a strong monotonic transverse wakefield along the full bunch length. Cylindrical or planar geometries may be used without altering the principle and the longitudinal beam profile reconstruction algorithm. In the first case an adjustable gap may be used to easily tune the wakefield amplitude, increasing the flexibility during the measurement. Nevertheless the intensity of the wakefield is reduced by a factor of $\pi^{2} / 16$ compared to the cylindrical geometry for a given aperture [26]. In the presented proof-of-principle experiment, at the maximum offset, the streaked beam size at the screen was less than a factor 4 longer than the trace of the unstreaked beam. This was sufficient to retrieve the bunch duration and roughly the electron beam shape. A high resolution measurement would require a much longer streaking compared to the unstreaked beam size at the screen. For an extensive use in $\mathrm{X}$-ray FEL machines, a passive streaker should be capable of time-resolving ultrashort bunches at high energies. A simulated passive streaking with a $2 \mathrm{~m}$ long corrugated structure shows the possibility of time-resolving a $200 \mathrm{fs}$ electron bunch at an energy $4 \mathrm{GeV}$, seeing details in the bunch structure on the attosecond scale [27]. Defocusing becomes less relevant for a small transverse size at the passive streaker achievable at high energies with low emittance beam by choosing properly the optics. Moreover defocusing effects can be compensated with a pair of orthogonal planar structures [14]. The absence of a bunchindependent time calibration is indeed a disadvantage but it can be mitigated by a fast reconstruction software and computing power for the tune-up. Single-shot nondestructive photon shape measurements [9] are the most important time-resolved electron bunch measurement in an X-ray FEL. These measurements provide necessary information to interpret correctly the experimental data, especially when double pulses are generated in the undulator line [28,29]. For this application the current profile is stable from shot to shot, thus the time calibration has to be done only once. Losses due to the longitudinal wakes are naturally taken into account when evaluating the difference between 
"lasing off" and "lasing on" images. The intrinsic poor resolution on the bunch head is unimportant when a spoiling technique is used to control the pulse duration [30]. Similarly, electrons on the bunch head do not lase in machines based on seeding, when the overlap between seed and electrons occurs only on the bunch core $[31,32]$.

[1] G. Berden, S. P. Jamison, A. M. MacLeod, W. A. Gillespie, B. Redlich, and A. F. G. van der Meer, Phys. Rev. Lett. 93, 114802 (2004).

[2] P. Kung, H.-c. Lihn, H. Wiedemann, and D. Bocek, Phys. Rev. Lett. 73, 967 (1994).

[3] S. Casalbuoni, B. Schmidt, P. Schmüser, V. Arsov, and S. Wesch, Phys. Rev. ST Accel. Beams 12, 030705 (2009).

[4] A. Lumpkin, B. Yang, and Y. Chae, Nucl. Instrum. Methods Phys. Res., Sect. A 393, 50 (1997), free Electron Lasers 1996.

[5] R. Akre, L. Bentson, P. Emma, and P. Krejcik, in Proceedings of the Particle Accelerator Conference, Chicago, IL, 2001 (IEEE, New York, 2001).

[6] D. Alesini, G. Di Pirro, L. Ficcadenti, A. Mostacci, L. Palumbo, J. Rosenzweig, and C. Vaccarezza, Nucl. Instrum. Methods Phys. Res., Sect. A 568, 488 (2006).

[7] M. Röhrs, C. Gerth, H. Schlarb, B. Schmidt, and P. Schmüser, Phys. Rev. ST Accel. Beams 12, 050704 (2009).

[8] P. Emma et al., Nat. Photonics 4, 641 (2010).

[9] C. Behrens et al., Nat. Commun. 5, 3762 (2014).

[10] S. Antipov, S. Baturin, C. Jing, M. Fedurin, A. Kanareykin, C. Swinson, P. Schoessow, W. Gai, and A. Zholents, Phys. Rev. Lett. 112, 114801 (2014).

[11] P. Emma, M. Venturini, K. L. F. Bane, G. Stupakov, H.-S. Kang, M. S. Chae, J. Hong, C.-K. Min, H. Yang, T. Ha, W. W. Lee, C. D. Park, S. J. Park, and I. S. Ko, Phys. Rev. Lett. 112, 034801 (2014).

[12] S. Bettoni, P. Craievich, M. Pedrozzi, and S. Reiche, in Proceedings, 35th International Free Electron Laser Conference (FEL 2013) (JACOW, NY, USA, 2013), p. 214.
[13] P. Craievich, Phys. Rev. ST Accel. Beams 13, 034401 (2010).

[14] F. Fu et al., Phys. Rev. Lett. 114, 114801 (2015).

[15] S. Antipov, C. Jing, M. Fedurin, W. Gai, A. Kanareykin, K. Kusche, P. Schoessow, V. Yakimenko, and A. Zholents, Phys. Rev. Lett. 108, 144801 (2012).

[16] M. Pedrozzi et al., in Proceedings, 33rd International Free Electron Laser Conference (FEL 2011) (JACOW, Shanghai, China, 2011), p. 625.

[17] W. Gai, P. Schoessow, B. Cole, R. Konecny, J. Norem, J. Rosenzweig, and J. Simpson, Phys. Rev. Lett. 61, 2756 (1988).

[18] K.-Y. Ng, Phys. Rev. D 42, 1819 (1990).

[19] W. Gai, J. G. Power, and L. Xiao, in Proceedings of the Particle Accelerator Conference, Chicago, IL, 2001 (IEEE, New York, 2001).

[20] K. L. F. Bane and G. V. Stupakov, in Proceedings, 20th International Linac Conference (LINAC 2000) (SLAC, Monterey, 2000).

[21] G. Stupakov, AIP Conf. Proc. 496, 341 (1999).

[22] A. V. Burov and A. V. Novokhatski, Institute for Nuclear Physics Technical Report No. BUDKERINP-90-28, 1990.

[23] K.-Y. Ng, Part. Accel. 25, 153 (1990).

[24] M. Borland, ANL Technical Report No. LS-287, 2000.

[25] P. Craievich, R. Ischebeck, F. Loehl, G. L. Orlandi, and E. Prat, in Proceedings, 35th International Free Electron Laser Conference (FEL 2013) (JACOW, NY, USA, 2013), p. 236.

[26] K. L. F. Bane and G. Stupakov, Phys. Rev. ST Accel. Beams 6, 024401 (2003).

[27] A. Novokhatski, Phys. Rev. ST Accel. Beams 18, 104402 (2015).

[28] A. Marinelli et al., Nat. Commun. 6, 6369 (2015).

[29] Y. Ding, C. Behrens, R. Coffee, F.-J. Decker, P. Emma, C. Field, W. Helml, Z. Huang, P. Krejcik, J. Krzywinski, H. Loos, A. Lutman, A. Marinelli, T. J. Maxwell, and J. Turner, Appl. Phys. Lett. 107, 191104 (2015).

[30] P. Emma, K. Bane, M. Cornacchia, Z. Huang, H. Schlarb, G. Stupakov, and D. Walz, Phys. Rev. Lett. 92, 074801 (2004).

[31] E. Allaria et al., Nat. Photonics 6, 699 (2012).

[32] E. Allaria et al., Nat. Photonics 7, 913 (2013). 